Check for updates

Cite this: RSC Adv., 2017, 7, 38149

Received 9th April 2017

Accepted 12th July 2017

DOI: $10.1039 / c 7 r a 04026 a$

rsc.li/rsc-advances

\title{
Development of nutrient media to increase the accumulation of lipids without genetic modification of a lipogenic microorganism
}

\begin{abstract}
Subhasish Das, (D) *ab Maria Alice Zarur Coelho, ${ }^{\text {P }}$ Priscilla F. F. Amaral ${ }^{a}$ and Jayeeta $\mathrm{Sil}^{a}$
Yarrowia lipolytica has drawn attention from the scientists over the last few decades because of its potential to accumulate intracellular lipid that can be transesterified to methyl esters with properties resembling those of diesel. A kinetics study of intracellular lipid production in Y. lipolytica is very important because it would help process modeling and cost minimization for increasing the lipid production. A set of defined media with varying amounts of pure glycerol as the carbon source and a fixed amount of ammonium sulfate as the nitrogen source and other media components, including thiamine and uracil supplements, was used to study the kinetics of lipid production. With an increase in the carbon to nitrogen $(C / N)$ ratio, lipid accumulation increased to a certain limit up to $4 \mathrm{~g} \mathrm{~L}^{-1}$, followed by substrate inhibition. The best rate and amount of lipid accumulation was predicted to be obtained in a cultivation medium with an initial glycerol concentration of $93 \mathrm{~g} \mathrm{~L}^{-1}$. While lipid production was high, citric acid production in the media was negligible. Minimization of citric acid synthesis via proper media development with nitrogen starvation and unique supplement utilization can increase lipid accumulation in Y. lipolytica IMUFRJ 50682 up to a great extent in mere batch cultivation without any genetic modification.
\end{abstract}

\section{Introduction}

Due to the ever increasing demand of petro-diesel and the ongoing depletion of petroleum resources, a major part of biochemical engineering research is focused on finding alternative sources of biofuel. Hence, bio-oil or lipid producing microorganisms have become a centre of research in recent decades. ${ }^{1,2}$ Yarrowia lipolytica is one such microorganism that is capable of accumulating lipid inside the cell as well as citric acid production. This yeast contains the ATP:citrate lyase (ACL) enzyme, which cleaves citrate that comes out of the mitochondria into the cytoplasm and thereby produces acetyl CoA in large quantities, which is a precursor for fatty acid synthesis. ACL is absent in Saccharomyces cerevisiae; hence, this microorganism cannot produce lipids. Moreover, Y. lipolytica contains malic enzyme, which converts malate to pyruvate along with the generation of reducing power (NADPH) for fatty acid synthesis. $^{3-6}$ Lipid accumulated in this yeast is considered as ideal for transesterification to produce biodiesel. In general, its major fatty acid composition is as follows: $7-23.1 \%$ of palmitic acid (16:0), $47.1-68.3 \%$ of stearic acid (18:0), $6.9-18.2 \%$ of oleic acid (18:1), and $2.2-8.9 \%$ of linolenic acid (18:2). ${ }^{3,4,7}$ Research has been carried out in many directions, including molecular

${ }^{a}$ Department of Biochemical Engineering, Federal University of Rio de Janeiro, Brazil ${ }^{b}$ Triphase Pharmaceuticals Pvt. Ltd., Mysore - 570016, India. E-mail: sdas@ triphasepharma.com; Tel: +91-821-4191022 modification of the genes involved in lipid metabolism, to increase the lipid accumulation capacity of this yeast. ${ }^{8-10}$ The leucine and uracil-supplemented complex medium for the leucine $^{+}$uracil $^{+}$strain of $Y$. lipolytica can produce high intracellular lipid, nearly $90 \%$ of the dry biomass. ${ }^{10}$ However, literature on the metabolic modification of Y. lipolytica without genetic modification and cultivation with glycerol is rare.

Although glycerol alone can support the growth of yeast to some extent, ${ }^{11}$ addition of supplements can improve the production of lipid and citric acid along with biomass growth., In this study, Y. lipolytica IMUFRJ 50682 was chosen because it could produce good amounts of citric acid as well as lipid to some extent. ${ }^{3,5}$ Yarrowia lipolytica (IMUFRJ 50682) is a wild-type strain isolated from an estuary in the vicinity of Rio de Janeiro, Brazil (Haegler and Mendonça-Haegler, 1981). It was identified by the Instituto de Microbiology of the Health Sciences Center of the Federal University of Rio de Janeiro. ${ }^{12,13}$ Glycerol as a carbon substrate and ammonium sulfate as a nitrogen source were used instead of glucose (C) and yeast extract (N) because of the following reasons. (1) Lipids are essentially triglycerides of long chain fatty acids. Therefore, it is hypothesized that if $Y$. lipolytica can consume glycerol as a carbon source, the yield of lipid should increase since the forward reaction should accelerate. Lipid synthesis using glycerol as a carbon source occurs directly through the formation of glycerol-3-phosphate, ${ }^{3,9}$ requiring less metabolic energy as compared to glucose metabolism. (2) Catabolism of lipids produces free fatty acids, 
such as citric acid, ${ }^{3,5,9}$ as a valuable by-product. (3) Glycerol is abundantly available as a by-product of biodiesel production via transesterification of vegetable oil., ${ }^{\mathbf{1 , 2 , 1 4}}$ (4) Complex media components, such as yeast extract, in the cultivation media for culturing yeast ${ }^{5,6}$ can act as a sole carbon source, nitrogen source and source of vitamins and minerals. These components would cause confusion while drawing any definitive conclusion over the experimental results obtained from this kinetic study. (5) Production of microbial lipid for obtaining biodiesel using lipid (e.g., industrial fats and vegetable oil) as a carbon source $^{15,16}$ may not always be an attractive strategy in the emerging biofuel industry.

Herein, the effects of $\mathrm{C} / \mathrm{N}$ ratio in a defined medium with varying initial concentrations of glycerol (as a carbon source) and ammonium salt (as a nitrogen source), along with thiamine and uracil supplementation, on lipid and citric acid production by Yarrowia lipolytica IMUFRJ 50682 have been studied.

\section{Experimental}

\section{Media composition}

A defined media ${ }^{3,17,18}$ was chosen for studying the effect of the C/ $\mathrm{N}$ ratio on $Y$. lipolytica IMUFRJ 50682. Pure glycerol with varying concentrations $\left(\mathrm{g} \mathrm{L}^{-1}\right)$ of $1.33,2.65,6.63,13.26,19.89,26.52$, 33.16, 42.44, 53.05, 66.31, 92.83, 103.05, 132.62, 172.41, 200, and 265.24 were used as the carbon source and ammonium sulfate $\left(3 \mathrm{~g} \mathrm{~L}^{-1}\right)$ was used as the only nitrogen source. As a vitamin source, only thiamine hydrochloride $\left(0.3 \mathrm{mg} \mathrm{L}^{-1}\right)$ was used along with uracil $\left(20 \mathrm{mg} \mathrm{L}^{-1}\right)$ as a supplement.

A $24 \mathrm{~h}$ culture of $Y$. lipolytica IMUFRJ 50682 in GPY $(2 \%$ glucose, $2 \%$ peptone, and $1 \%$ yeast extract) medium was used as inoculum, from which about $10^{9}$ cells $(1 \%$ of the defined medium) were inoculated in different conical flasks containing defined media with different concentrations of pure glycerol as abovementioned. The flasks were incubated in a shaker incubator under the following conditions: shaker speed $200 \mathrm{rpm}$, temperature $28{ }^{\circ} \mathrm{C}$.

\section{Analytical methods}

At regular intervals, $1 \mathrm{~mL}$ samples were withdrawn for the following analyses: biomass concentration (dry weight as well as $\mathrm{OD}_{600 \mathrm{~nm}}$ ), lipid droplet index (LD index), citric acid concentration, residual glycerol concentration, and residual ammonium ion determination.

Citric acid concentration and residual glycerol concentration were measured using HPLC. ${ }^{\mathbf{1 4 , 1 9}}$

Lipid droplet indices of the samples were measured by a method described by Morais et al. ${ }^{20}$ using the BODIPY fluorescent dye. Lipid was extracted and quantified by the chloroform-methanol method. ${ }^{18,21}$ Residual $\left[\mathrm{NH}_{4}{ }^{+}\right]$concentration was measured using a Himedia soil testing kit. A calibration curve was made using varying known concentrations of ammonium sulphate, and the resulting brownish solution was assayed at $700 \mathrm{~nm}$ (where the absorption maxima was found).
Microscopic analysis was performed using a phase-contrast microscope (Nikon Eclipse E200, Japan) with a $100 \times 10$ magnification.

Lipid extracted from $Y$. lipolytica IMUFRJ 50682 was analyzed via gas-chromatographic analysis to study its composition. ${ }^{18}$

Product yield upon biomass $Y_{\mathrm{P} / \mathrm{X}}$ was calculated from the following equation:

$$
Y_{\mathrm{P} / \mathrm{X}}=\frac{P-P_{0}}{X-X_{0}}
$$

whereas product yield upon substrate $Y_{\mathrm{P} / \mathrm{S}}$ was calculated from the following equation:

$$
Y_{\mathrm{P} / \mathrm{S}}=\frac{P-P_{0}}{S_{0}-S}
$$

All terms in each equation represent conventional meanings.

\section{Results and discussion}

With different initial concentrations of glycerol, it was observed that there was variation in the growth and lipid production profile of the $Y$. lipolytica strain (Fig. 1). Increase in the initial glycerol concentration caused increase in the biomass and lipid yield (Fig. 1, Table 1). Lipid was produced in a semi-growth associated fashion. Citric acid concentration was too low to be detected in all the cultures. At low $S_{0}\left(1.64 \mathrm{~g} \mathrm{~L}^{-1}\right)$, the carbon source was totally consumed, but as much as $0.6 \mathrm{~g} \mathrm{~L}^{-1}$ ammonium sulphate remained unabsorbed from the culture medium by the yeast [Fig. 1]. In culture with $S_{0}=6.8 \mathrm{~g} \mathrm{~L}^{-1}$ glycerol, more nitrogen was consumed as compared to a culture with lower $S_{0}$ $\left(1.64 \mathrm{~g} \mathrm{~L}^{-1}\right)$. In culture with $S_{0}=42.4 \mathrm{~g} \mathrm{~L}^{-1}$ glycerol, almost complete consumption of nitrogen was observed over a longer period of time. With a glycerol concentration $S_{0}=92.83 \mathrm{~g} \mathrm{~L}^{-1}$, a small amount of nitrogen source $\left(0.2 \mathrm{~g} \mathrm{~L}^{-1}\right)$ remained unconsumed even after the maximum biomass growth and lipid production was complete. In cultures with lower $S_{0}$, glycerol was exhausted earlier than nitrogen along with an increase in biomass. Even after glycerol was left at too low concentration to be absorbed by the yeast cells, nitrogen concentration continued to decrease with a simultaneous decrease in intracellular lipid concentration. Similar results were also observed for higher $S_{0}\left(42.44 \mathrm{~g} \mathrm{~L}^{-1}\right.$ and $\left.92.83 \mathrm{~g} \mathrm{~L}^{-1}\right)$. Therefore, after reaching maxima, lipid concentration did not remain unchanged for rest of the cultivation period; this indicated lipid catabolism and led to a decrease in the total biomass in the culture (Fig. 1, Table 1).

Beyond $S_{0}=92.83 \mathrm{~g} \mathrm{~L}^{-1}$, lipid yield gradually decreased (Fig. 2) although there was no detectable citric acid in these cultures. This observation did not agree with previous literature ${ }^{6,7}$ where it was indicated that with the increasing $\mathrm{C} / \mathrm{N}$ ratio, initially a proportional increase in the biomass yield was observed up to certain $S_{0}$. Further increase in the $\mathrm{C} / \mathrm{N}$ ratio causes intracellular lipid accumulation as well as extracellular citric acid production, which keeps on increasing up to an optimum $\mathrm{C} / \mathrm{N}$ proportion, beyond which lipid accumulation decreases, but extracellular citric acid production keeps on 

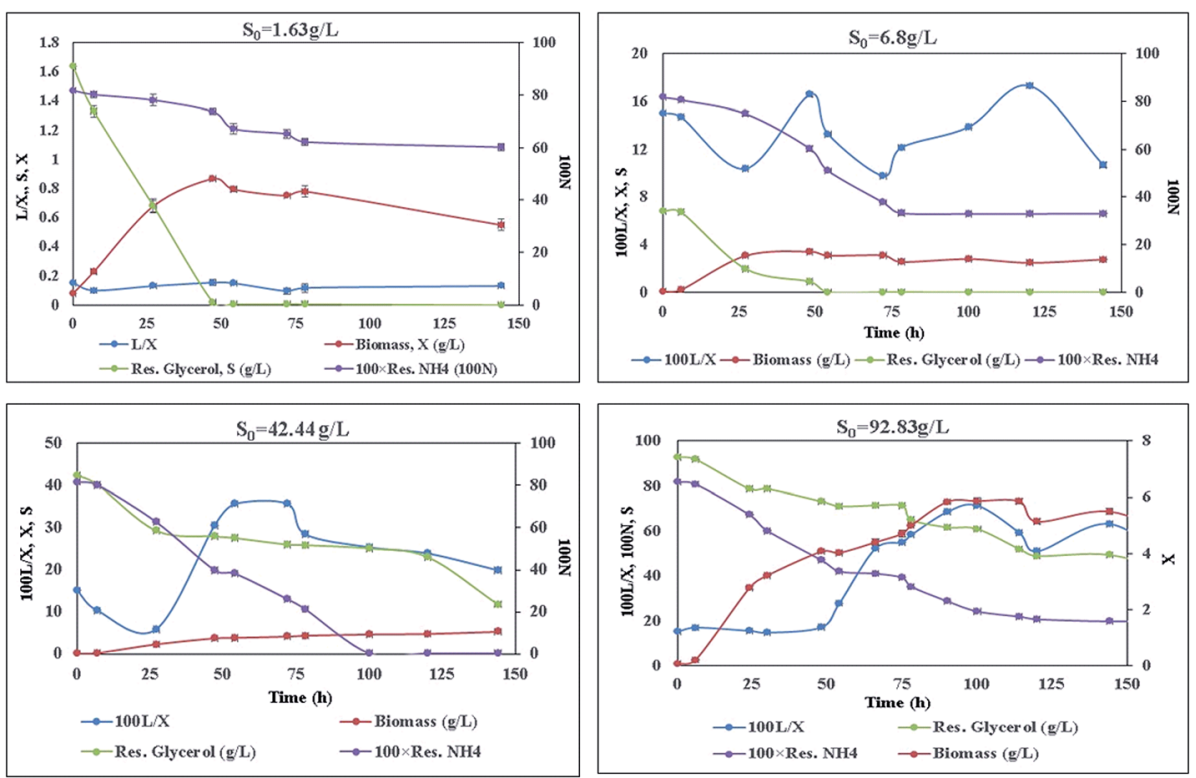

Fig. 1 Time course of total biomass in $\mathrm{g}^{-1}(\mathrm{X})$, lipid in $\mathrm{g}^{-1}(\mathrm{~L})$ and residual glycerol in $\mathrm{L}^{-1}(\mathrm{~S})$, residual ammonium ion in $\mathrm{g} \mathrm{L}^{-1}(\mathrm{~N})$ for broth with initial glycerol concentration $\left(S_{0}\right)$ of $1.63 \mathrm{~g} \mathrm{~L}^{-1}, 6.8 \mathrm{~g} \mathrm{~L}^{-1}, 42.44 \mathrm{~g} \mathrm{~L}^{-1}$, and $92.83 \mathrm{~g} \mathrm{~L}^{-1}$.

Table 1 Comparison of lipid yield in different cultures

\begin{tabular}{lllll}
\hline $\begin{array}{l}S_{0} \\
(\text { glycerol, g L }\end{array}$ & $\begin{array}{l}\text { Biomass } \\
\left(\mathrm{g} \mathrm{L}^{-1}\right)\end{array}$ & $\begin{array}{l}\text { Total lipid } \\
\left(\mathrm{g} \mathrm{L}^{-1}\right)\end{array}$ & $Y_{\mathrm{P} / \mathrm{S}}$ & $\begin{array}{l}\text { Time of } \\
\text { maximum yield }\end{array}$ \\
\hline 1.64 & & & & \\
6.80 & 0.87 & 0.13 & 0.072 & $48 \mathrm{~h}$ \\
42.44 & 3.42 & 0.57 & 0.094 & $48 \mathrm{~h}$ \\
92.83 & 4.11 & 1.47 & 0.089 & $72 \mathrm{~h}$ \\
& 5.89 & 4.17 & 0.104 & $100 \mathrm{~h}$
\end{tabular}

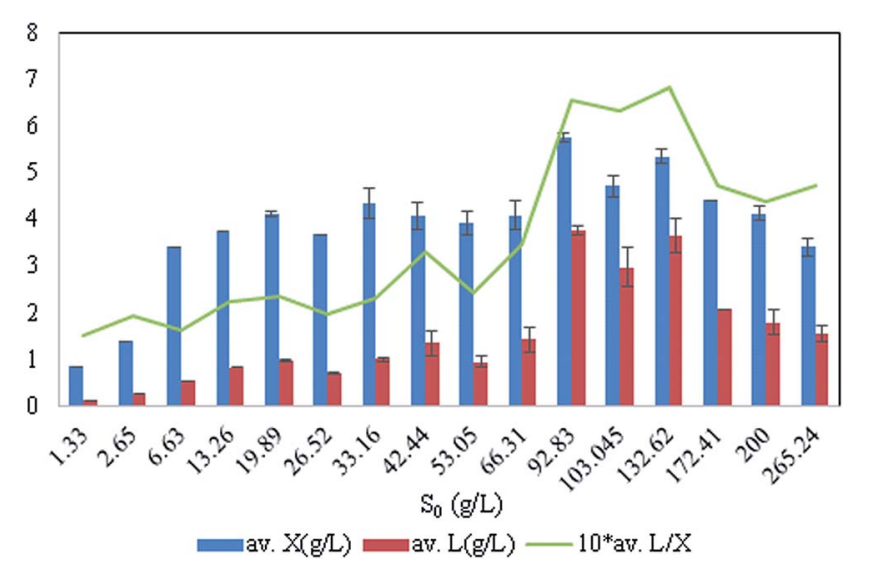

Fig. 2 Effect of initial glycerol concentration, $S_{0}$ (with fixed nitrogen concentration) in culture media on lipid $(\mathrm{L})$ and biomass $(\mathrm{X})$ yields.

increasing. Thiamine (a vitamin not synthesized by Y. lipolytica)-limited medium is known to help in the production of $\alpha$ ketoglutaric acid and pyruvic acid, whereas thiaminesupplemented medium is supposed to induce citric acid production at low $\mathrm{pH} .{ }^{3}$ Since $Y$. lipolytica IMUFRJ 50682 could produce as much as $8.64 \mathrm{~g} \mathrm{~L}^{-1}$ citric acid and $6.8 \%$ lipid upon biomass $^{5}$ in a complex medium containing glucose, yeast extract, and other nutrients, ${ }^{11}$ in this study, it was expected that in the defined medium, the yeast should produce both citric acid and lipid even though the yield may be lower than in the complex medium. The complex medium containing yeast extract and peptone was used for inoculum medium preparation, but production medium used in this study was thiaminesupplemented defined medium. Initially, within the first 6-12 hours, pH decreased below 3.5 in all cultures with different $S_{0}$ values and remained constant throughout likely because some or all of these organic acids were produced (data not shown in figure). There could hence be two possibilities: either citric acid excreted by the yeast cells was taken up as a carbon source, ${ }^{3}$ or citrate transporter proteins across cell membrane had been jeopardized. The first possibility does not seem to be practical as compared to the second possibility since in the first case, the cells have to spend more ATPs: once for excretion of citric acid and again for absorption of citric acid as a carbon source. Therefore, it is possible that inhibitors of citrate transport had been generated, which could be protein or peptide in nature. ${ }^{22}$ In the culture medium with $S_{0}=92.83 \mathrm{~g} \mathrm{~L}^{-1}$, it was observed in the culture samples at $24 \mathrm{~h}$ and $300 \mathrm{~h}$ that supernatant thiamine was intact (Fig. 3), whereas uracil was completely exhausted within the first few hours (not shown in figure). The effects of the supplements could be observed from the distinguishing cellular morphological features of $100 \mathrm{~h}$ old cultures of the yeast with supplements in the culture medium (Fig. 4A) and without supplements in the culture medium (Fig. 4B). With supplements, polar lipid accumulation in cell membranes was less prominent, indicating a need to synthesize cellular machinery to withstand physiological stress due to high glycerol concentration by exploiting the excess glycerol intake. 



Fig. 3 HPLC analysis of citric acid production \& thiamine consumption $\left(S_{0}=92.83 \mathrm{~g} \mathrm{~L}^{-1}\right)$.
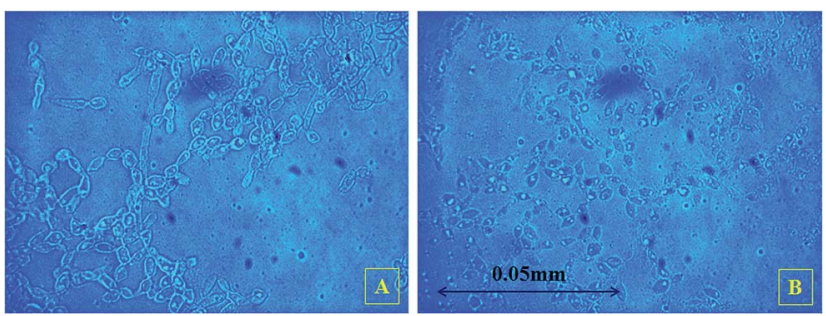

Fig. 4 Phase contrast images of the $100 \mathrm{~h}$ old samples (A) with supplements in culture medium and (B) without supplements in culture medium.

Media were prepared with and without thiamine and/or uracil (Table 2) to find out changes in the lipid, citric acid, and biomass yield, if any. However, total lipid yield did not decrease much, but citric acid yield was negligible. In fact, medium with thiamine and without uracil had a lipid yield of about $84 \%$ although the biomass yield decreased. Flask experiments do not generally produce much biomass because of oxygen limitation. Yarrowia lipolytica, being a strict aerobe, did not produce much biomass in the described experiments. However, it is to be noted that with increased oxygen supply, $\beta$ oxidation and catabolism of lipid is increased in Y. lipolytica (Alice et al. 2010). ${ }^{3}$ Therefore, oxygen limitation actually helped to reduce lipid loss in our study.

This also led us to conclusion that yeast extract contains certain factor(s) other than thiamine or uracil that stops excretion of citric acid from cells or decreases lipid metabolism in this yeast by increasing citric acid secretion by the yeast cells. Citrate obtained from the TCA cycle can either be excreted as citric acid or can start lipid biosynthesis by forming acetyl Co-A and using ATP:citrate lyase (ACL) and other enzymes. In the present defined media, lipid yield increased because blockage of the extracellular citric acid production led to enhanced lipid accumulation (Fig. 5) via the ATP:citrate lyase (ACL) pathway., ${ }^{3,4-10,23}$ As abovementioned, with genetic modification, lipid accumulation can increase by as much as $90 \%$ of oleaginous yeast biomass.${ }^{10}$ Our findings of over $70 \%$ lipid accumulation without genetic modification are really interesting in this context.

Lipid compostion analysis from IMUFRJ 50682 showed predominance of oleic acid $(46.8 \%)$, followed by palmitoleic acid (18\%), palmitic acid (13.5\%) (Table 3). A higher percentage of oleic acid in lipid would is desirable for biodiesel production. ${ }^{7,18}$ In this context it would be noteworthy to compare other reports on $Y$. lipolytica fatty acid compostion. Kamzolova et al. (2011) observed that the limitation of Y. lipolytica grown on

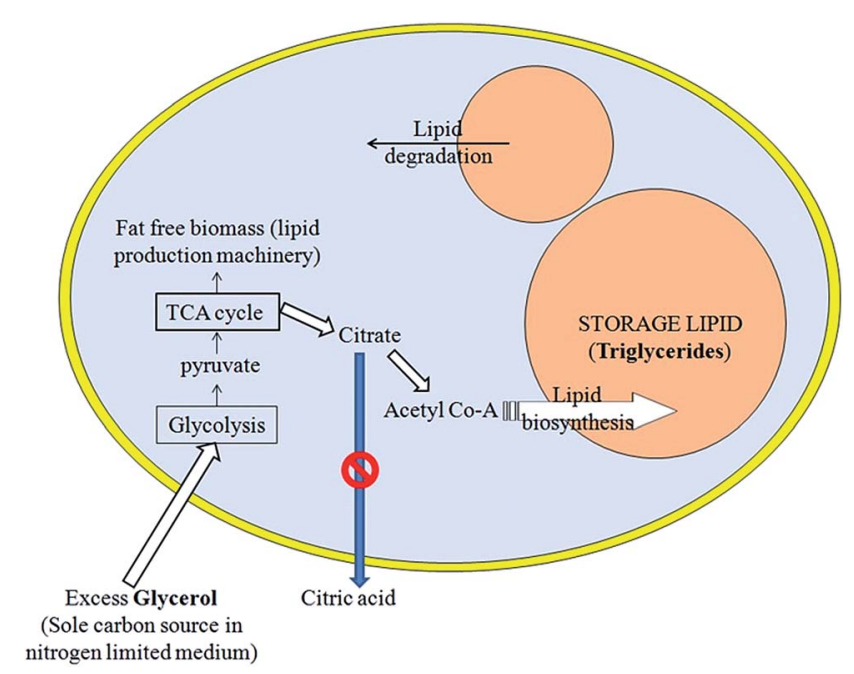

Fig. 5 Schematic showing how suppression of citrate excretion from yeast cells might cause excess lipogenesis.

Table 2 Effect of uracil and thiamine on biomass and lipid yield

\begin{tabular}{llcr}
\hline$S_{0}$ (glycerol, $\left.\mathrm{g} \mathrm{L}^{-1}\right)$ & Biomass $\left(\mathrm{g} \mathrm{L}^{-1}\right)$ & Total lipid $\left(\mathrm{g} \mathrm{L}^{-1}\right)$ & Time of maximum yield \\
\hline 92.83 (with uracil \& thiamine) & $5.87 \pm 0.12$ & $4.19 \pm 0.03$ & $100 \mathrm{~h}$ \\
92.83 (with thiamine, no uracil) & $5.21 \pm 0.33$ & $3.55 \pm 0.15$ & $100 \mathrm{~h}$ \\
92.83 (no supplement) & $5.84 \pm 0.10$ & $3.6 \pm 0.02$ & $100 \mathrm{~h}$
\end{tabular}


Table 3 Fatty acid composition of extracted lipid from Y. lipolytica IMUFRJ 50682

\begin{tabular}{llc}
\hline & Fatty acid (FA) & $\%(\mathrm{~g} / 100 \mathrm{~g}$ FA $)$ \\
\hline Pentadecanoic acid & C15:0 & 1.46 \\
Palmitic acid & C16:0 & 13.48 \\
Palmitoleic acid & C16:1 & 17.59 \\
Heptadecanoic acid $^{a}$ & C17:0 & 4.08 \\
Stearic acid & C18:0 & 3.66 \\
Oleic acid & C18:1 & 46.79 \\
Linoleic acid & C18:2 & 12.94 \\
${ }^{a}$ Internal standard. & &
\end{tabular}

glycerol-containing waste from biodiesel industry would result in biomass production enriched lipids ( $29.9 \%$ of dry biomass in growth phase). It was observed that the lipids of $Y$. lipolytica, grown on crude glycerol, were represented mainly by oleic, linoleic, palmitic and palmitoleic acids. But during the transition of citric acid-formation phase a significant alteration of the fatty acid composition happened; the content of oleic $(\Delta 9 \mathrm{C} 18: 1)$ and palmitic (C16:0) acids decreased from 61.8 to $56.8 \%$ and from 7.23 to $4.57 \%$, respectively, whereas the amount of palmitoleic acid ( $\triangle 9$ C16:1) increased from 1.8 to $9.03 \%$. The amount of linoleic acid $(\Delta 9,12 \mathrm{C} 18: 2)$ did not markedly change. High values of a C18:1/C18:0 ratio revealed in all variants were indicative of high activity of $\Delta 9$-desaturase, especially in the citric acid production phase. ${ }^{24}$ Another study revealed that the fatty acid composition of intracellular lipids in Y. lipolytica, grown on rapeseed oil was similar to that of rapeseed oil with predominance of oleic $(\Delta 9 \mathrm{C} 18: 1)$ and linoleic $(\Delta 9,12 \mathrm{C} 18: 2)$ acids ( 61.9 and $29.2 \%$ of the total fatty acids, respectively). ${ }^{25}$

Sara et al. (2016) observed the significant lipid could be produced (13.6 $\mathrm{g} \mathrm{L}^{-1}$ and lipid content $52.7 \%$ ) with wild strain $Y$. lipolytica grown on crude glycerol based media. They also reported that the yeast lipid contained mainly oleic, palmitic, linoleic and stearic acids and indicated that this lipid would be ideal for the synthesis of biodiesel. ${ }^{7}$

\section{Conclusion}

The novelty of findings obtained in this study are as follows: increased intracellular lipid accumulation by developing proper cultivation media, without genetic modification of the lipogenetic microorganism ( $Y$. lipolytica). Certain nutritional factors (other than thiamine and uracil) present in yeast extract activate genes involved in extracellular citric acid production. Without this factor in the culture medium, $Y$. lipolytica can channelize citrate into the lipid biosynthesis pathway.

\section{Acknowledgements}

Subhasish Das acknowledges CNPq, Brazil, for funding (Processo: 503313/2011-4) and students of Room No. E-103, Escola de Quimica, UFRJ for helping in analytical methods. M. A. Z. Coelho and P. F. F. Amaral thanks both CNPq and PETROBRAS for project funding.

\section{References}

1 M. R. J. Almeida, L. C. L. Fávaro and F. B. Quirino, Biodiesel biorefinery: opportunities and challenges for microbial production of fuels and chemicals from glycerol waste, Biotechnol. Biofuels, 2012, 5, 48.

2 F. Yang, A. M. Hanna and R. Sun, Value-added uses for crude glycerol - a byproduct of biodiesel production, Biotechnol. Biofuels, 2012, 5, 13.

3 M. A. Z. Coelho, P. F. F. Amaral and I. Belo, Yarrowia lipolytica: an industrial workhouse, Current Research, Technology and Education Topics in Applied Microbiology and Microbial Biotechnology, ed. A. Mendez-Vilas, FORMATEX, 2010.

4 A. Makri, S. Fakas and G. Aggelis, Metabolic activities of biotechnological interest in Yarrowia lipolytica grown on glycerol in repeated batch cultures, Bioresour. Technol., 2010, 101, 2351-2358.

5 J. Sil, S. Das, R. G. Oliveira, P. F. F. Amaral and M. A. Z. Coelho, Screening six potential Yarrowia lipolytica strains for best lipid, citric acid, biosurfactant and lipase production, Proceedings of 2013 International Conference on Environment, Energy and Biotechnology (CBEES 2013), Kuala lumpur, 8-9 June 2013, pp. 117-121, DOI: 10.7763/ ipcbee.2013.v51.22.

6 S. Papanikolaou, L. Muniglia, I. Chevalot, G. Aggelis and I. Marc, Yarrowia lipolytica as a potential producer of citric acid from raw glycerol, J. Appl. Microbiol., 2002, 92, 737-744.

7 M. Sara, S. K. Brar and J. F. Blais, Lipid production by Yarrowia lipolytica grown on biodiesel-derived crude glycerol: optimization of growth parameters and their effects on the fermentation efficiency, RSC Adv., 2016, 6(93), 90547-90558.

8 M. Rossi, A. Amaretti, S. Raimondi and A. Leonardi, Getting lipids for biodiesel production from oleaginous fungi, in Biodiesel - Feedstocks and Processing Technologies, ed. M. Stoytcheva and G. Montero, InTech, Rijeka, Croatia, 2011, ch. 4, pp. 71-92.

9 A. Beopoulos, J. Cescut, R. Haddouche, L. J. Uribelarrea, M. C. Jouve and M. J. Nicaud, Yarrowia lipolytica as a model for bio-oil production, Prog. Lipid Res., 2009, 48, 375-387.

10 J. Blazeck, A. Hill, L. Liu, R. Knight, J. Miller, A. Pan, P. Otoupal and H. S. Alper, Harnessing Yarrowia lipolytica lipogenesis to create a platform for lipid and biofuel production, Nat. Commun., 2014, 5, 3131, DOI: 10.1038/ ncomms4131.

11 S. Swinnen, M. Klein, M. Carrillo, J. McInnes, T. H. Nguyen and E. Nevoigt, Re-evaluation of glycerol utilization in Saccharomyces cerevisiae: characterization of an isolate that grows on glycerol without supporting supplements, Biotechnol. Biofuels, 2013, 6, 157, DOI: 10.1186/1754-6834-6-157.

12 F. V. Nascimento, P. F. F. Amaral and M. A. Z. Coelho, Evaluation of erythritol production by yeast Yarrowia lipolytica IMUFRJ 50682 using experimental design, XXI 
Brazilian Congress of Chemical Engineering, September 2016, pp. 25-29, ENBEQ 2016.

13 P. F. F. Amaral, M. Lehocky, A. M. V. Barros-Timmons, M. H. M. Rocha-Leão, M. A. Z. Coelho and J. A. P. Coutinho, Cell surface characterization of Yarrowia lipolytica IMUFRJ 50682, Yeast, 2006, 23, 867-877.

14 L. V. Silva, B. T. Camila, P. F. F. Amaral and M. A. Z. Coelho, Production of citric acid by Yarrowia lipolytica in different crude glycerol concentrations and in different nitrogen sources, Chemical Engineering Transactions, 2012, 27, 199-204.

15 S. Papanikolaou and G. Aggelis, Modeling lipid accumulation and degradation in Yarrowia lipolytica cultivated on industrial fats, Curr. Microbiol., 2003, 46, 398-402.

16 G. Aggelis and J. Sourdis, Prediction of lipid accumulationdegradation in oleaginous micro-organisms growing on vegetable oils, Antonie Van Leeuwenhoek, 1997, 72, 159-165.

17 N. Gasmi, A. Ayed and H. N. M. J. Kallel, Design of an efficient medium for heterologous protein production in Yarrowia lipolytica: case of human interferon alpha $2 \mathrm{~b}$, Microb. Cell Fact., 2011, 10, 38.

18 J. Chopra, R. Dineshkumar, M. Bhaumik, G. Dhanarajan, R. R. Kumara and R. Sen, Integrated in situ transesterification for improved biodiesel production from oleaginous yeast: a value proposition for possible industrial implication, $R S C$ Adv., 2016, 6, 70364.

19 L. V. Silva, C. B. Tavares, P. F. F. Amaral and M. A. Z. Coelho, Production of citric acid by Yarrowia lipolytica in different crude glycerol concentrations and in different nitrogen sources, Chemical Engineering Transactions, 2012, 27, 199-204.
20 B. L. B. Morais, J. B. Madeira, M. C. M. Monteiro, C. A. Masuda and M. M. Lomeli, A New FluorescenceBased Method Identifies Protein Phosphatases Regulating Lipid Droplet Metabolism, PLoS One, 2010, 5(10), e13692, DOI: 10.1371/journal.pone.0013692.

21 S. J. Lee, B.-D. Yoon and H.-M. Oh, Rapid method for determination of lipid from green alga Botryococcus braunii, Biotechnol. Tech., 1998, 12, 553-556.

22 J. Sun, S. Aluvila, R. Kotaria, J. A. Mayor, D. E. Walters and R. S. Kaplan, Mitochondrial and plasma membrane citrate transporters: discovery of selective inhibitors and application to structure/function analysis, Mol. Cell. Pharmacol., 2010, 2(3), 101-110.

23 G. Christophe, V. Kumar, R. Nouaille, G. Gaudet, P. Fontanille, A. Pandey, R. C. Soccol and C. Larroche, Recent Developments in Microbial Oils Production: a Possible Alternative to Vegetable Oils for Biodiesel Without Competition with Human Food?, Braz. Arch. Biol. Technol., 2012, 55, 29-46.

24 S. V. Kamzolova, A. R. Fatykhova, E. G. Dedyukhina, S. G. Anastassiadis, N. P. Golovchenko and I. G. Morgunov, Citric acid production by yeast grown on glycerolcontaining waste from biodiesel industry, Food Technol. Biotechnol., 2011, 49, 65-74.

25 S. V. Kamzolova, E. G. Dedyukhina, V. A. Samoilenko, J. N. Lunina, I. F. Puntus, R. K. Allayarov, M. N. Chiglintseva, A. A. Mironov and I. G. Morgunov, Isocitric acid production from rapeseed oil by Yarrowia lipolytica yeast, Appl. Microbiol. Biotechnol., 2013, 97, 9133-9144. 